Project 1022519

\title{
Integrated Investigation on the Production and Fate of Organo-Cr(III) Complexes from Microbial Reduction of Chromate
}

\author{
Xun, Luying \\ Washington State University
}

RESULTS TO DATE: Progress and immediate actions for each task are summarized here.

Task 1. Production of soluble organo-Cr(III) complexes by selected microorganisms

The screening of different genera of bacteria for production of soluble $\mathrm{Cr}$ (III) complexes has been completed. A total of eight organisms were screened for production of soluble $\mathrm{Cr}(\mathrm{III})$; three were Gram positive and five were Gram negative. The Gram positive bacteria were Cellulomonas sp. ES 6, Rhodococcus sp., and Leafsonia sp., while Shewanella. oneidensis MR 1, Desulfovibrio desulfuricans G20, D. vulgaris Hildenborough, Pseudomonas putida MK 1 and Ps. aeruginosa PAO 1 were Gram negative. S. oneidensis MR 1 and Cellulomonas sp ES 6 were grown in minimal media, GWM (Ground Water Medium with lactate/fumarate) and SGM ( Simulated Groundwater Medium with sucrose), respectively. Other bacteria were screened under non-growth conditions with sucrose, lactate, or glycerol as electron donor. All experiments were carried out for a period of 15-30 days, with different organisms reaching a maximum soluble $\mathrm{Cr}(\mathrm{III})$ concentrations at different times: S. oneidensis, 2d; Cellulomonas sp., 8d; Leafsonia, 6d; Rhodococcus, 9d; Ps. putida MK 1, 6d, Ps. aeruginosa PAO 1, 3d; D. vulgaris Hildenborough, 3d; and D. desulfuricans G20, 21d. Initial characterization indicates that the soluble $\mathrm{Cr}$ (III) fraction produced by both S. oneidensis MR 1 and Cellulomonas sp. ES 6 passes through a 1-Kd cut off filter. Thus the $\mathrm{Cr}(\mathrm{III})$ does not appear to be protein bound under these conditions. The soluble $\mathrm{Cr}$ complex of S. oneidensis MR 1 was enriched by lyophilization followed by solvent extraction. After this purification about $12.5 \%$ of the soluble $\mathrm{Cr}$ was soluble in methanol, and about $60 \%$ of the complex was soluble in water. The water soluble fraction was retained on an anion exchange column and could be eluted by a salt solution, indicating the $\mathrm{Cr}(\mathrm{III})$ is in a complex with negative charges. Further characterization of this compound is underway, and a similar approach will be attempted to characterize the soluble $\mathrm{Cr}(\mathrm{III})$ complexes produced by the other bacteria.

We have been developing techniques for characterizing the organo- $\mathrm{Cr}(\mathrm{III})$ complexes. We have developed a method of mass spectrometry to enable us to characterize $\mathrm{Cr}$ (III) complexes with simple organic ligands. With our method, we are attempting to characterize the complexation of $\mathrm{Cr}$ (III) with gluconate, ascorbate, and NADH. Once this method is fully developed, then we will attempt to evaluate the organo- $\mathrm{Cr}(\mathrm{III})$ complexes produced during microbial reduction.

We are also developing electron paramagnetic resonance spectrometry (EPR) methods to follow the oxidation state of the $\mathrm{Cr}$ during reduction from chromate to $\mathrm{Cr}(\mathrm{III})$. Recent work by others has indicated the possibility of identifying intermediates such as $\mathrm{Cr}(\mathrm{V})$ during reduction to $\mathrm{Cr}(\mathrm{III})$. Although this oxidation state is only present as an intermediate, it may play a role in formation of the final organo- $\mathrm{Cr}(\mathrm{III})$ complexes.

Task 2. Demonstrate that chromate reduction produces organo- $\mathrm{Cr}$ (III) complexes with microbial cellular components. Research focused on the production of soluble organo- $\mathrm{Cr}(\mathrm{III})$ complexes has proceeded using a method of reducing $5 \mathrm{mM} \mathrm{Cr}(\mathrm{VI})$ in the presence of $20 \mathrm{mM}$ organic in a highly buffered solution at neutral $\mathrm{pH}, 100 \mathrm{mM}$ Potassium Phosphate $(\mathrm{pH} \mathrm{7})$. We have tested for the formation of soluble organo$\mathrm{Cr}$ (III) complexes using 18 different organic components, amino acids, TCA cycle intermediates, etc. Thus far we have identified 10 highly soluble organo- $\mathrm{Cr}$ (III) complexes, 2 slightly soluble complexes with both soluble and insoluble $\mathrm{Cr}(\mathrm{III})$, and 6 that form mainly insoluble $\mathrm{Cr}$ (III) complexes. Five different highly soluble complexes have been analyzed by UV/vis spectrometry and EPR. Results of the UV/vis analysis showed a red shifting of the $\mathrm{Cr}$ (III) peak in the 580 - 600nm range. Similar results were noted for the 
previously published NAD+-Cr(III) complexes and ascorbate- $\mathrm{Cr}$ (III) complexes. EPR analysis gave peaks with much broader curves in comparison to the monomeric $\mathrm{Cr}(\mathrm{III})$ control. The broad spectra were again similar to both the NAD+- $\mathrm{Cr}$ (III) and ascorbate $\mathrm{Cr}$ (III) complexes. Thus far the results indicate that soluble organo- $\mathrm{Cr}$ (III) complexes readily form in the presence of cellular organics. Further experiments to characterize the complexes based on the organic to $\mathrm{Cr}$ (III) ratio of the complex, molecular weight by mass spectrometry, and identifying the specific ligands involved in binding the organic portion to the $\mathrm{Cr}$ (III) ion will be performed.

Task 3. investigate the biological and abiotic transformation of organo- $\mathrm{Cr}$ (III) complexes formed during microbial reduction of chromate.

Investigation into the biological transformation of $\mathrm{NAD+-Cr(III)}$ complexes by the gram-positive bacterium has thus far identified that NAD+ is the carbon source for growth. This has been confirmed by repeated growth experiments using NAD+- $\mathrm{Cr}(\mathrm{III})$ complexes as the sole carbon and energy source in solution, and by growing the bacterium on a minimal medium using NAD+ as the sole carbon and energy source. Further characterization of the insoluble $\mathrm{Cr}(\mathrm{III})$ after NAD+ consumption by the bacterium will be performed by transmission electron microscopy coupled with an electron X-ray dispersive spectrophotometer, to gain a better understanding of the nature of the $\mathrm{Cr}$ (III) precipitate. Preliminary phylogenetic analysis of the $16 \mathrm{~s}$ rDNA identifies the bacterium as belonging to the Leifsonia genus, which are gram-positive soil aerobes. Future experiments to characterize the biochemical and metabolic profiles of the bacterium will be completed along with screening the bacterium for mineralization of other organo-Cr(III) complexes.

Task 4. Transport and fate of organic-Cr(III) complexes in soil. Batch equilibrium soil sorption experiments using artificially produced organo $\mathrm{Cr}$ (III) complexes ( $\mathrm{Cr}(\mathrm{III})$-serine, -cysteine and -malate) will be performed. These experiments will be followed up with soil sorption studies of the purified compounds obtained directly from bacterial cultures. This task was delayed as efforts were focused on Task 1, 2, and 3 for the first year.

DELIVERABLES: We have not published any papers, yet. We have two manuscripts in preparation. 